\title{
Collaboration: A potential solution to imminent issues facing nursing and midwifery education in Africa?
}

The Year of the Nurse and Midwife will dominate the academic discourse for years to come. There was no better way to celebrate and elevate the status of nursing and midwifery globally than a true demonstration of the work of nurses and midwives during the COVID-19 pandemic. In addition, the first ever State of the World's Nursing Report was released this year, highlighting milestones in the nursing profession and also the glaring challenges in nursing, nursing education and health systems in general. ${ }^{[1]}$ As we look into the future, I reflect on some of the imminent issues facing nursing and midwifery education on the African continent.

The contribution to nursing theory by nurses from Africa is still debatable. A link between nursing theory and the formation of professional identity has been established. ${ }^{\left[{ }^{2]}\right.} \mathrm{A}$ well-formed professional identity enhances the contribution of nurses to healthcare, and challenges the dominant norms of nurses being 'hand-maidens' who act on instructions from other health professionals. Basing nursing education on contextually developed nursing models and theory supports the formation of an appropriate professional identity among graduating nurses and midwives. It is crucial for the future of nursing education in Africa that models and theories related to nursing are developed from, and also reflect realities on, the African continent.

The World Health Organization (WHO) has promulgated interprofessional education as fundamental for the development of collaborative practiceready health professionals. ${ }^{[3]}$ Interprofessional education enhances students' awareness of other health professionals' roles, and improves communication and teamwork. ${ }^{[4]}$ Face-to-face institution-based interprofessional education initiatives have since been reported in Africa, involving nursing students in university-based programmes. The literature is silent on interprofessional education opportunities for nursing students in non-university-based programmes, regardless of them being the largest number of nursing students on the continent. With the rise of online and electronic approaches to learning and teaching, virtual interprofessional education may be an alternative. Such education may provide an opportunity to include students from various settings, e.g. nursing students in non-universitybased programmes.

There are renewed calls for the adoption and implementation of competency-based nursing education across all regions. ${ }^{[1]}$ Several reports from the continent have highlighted structural challenges related to the adoption of competency-based education. ${ }^{[5]}$ Nursing education institutions seem to have limited support from stakeholders for any meaningful curriculum change. The lack of support for these institutions may potentially negatively influence the quality of nursing education, thus doing more harm than the intended good.

Nurse educators are vital in the development of future nurses. In Africa, in addition to the perpetual shortage of nurse educators, the majority of them are not involved in research and academic scholarship. Furthermore, emerging nurse educators grapple with research methodology, resulting in poor-quality research, with limited application beyond a specific context. Ultimately, this situation restricts any meaningful contribution to nursing science by nurse educators from the African continent.

This special focus issue on nursing and midwifery education in Africa reflects the collaboration among nurses and non-nursing professionals from different institutions within a country and across Africa. These examples of collaboration and network-building outline some of the ways to overcome parochial concerns and embrace the context of our practice.

\section{Champion N Nyoni}

Guest editor: Special focus issue on nursing and midwifery education, School of Nursing, Faculty of Health Sciences, University of the Free State, Bloemfontein, South Africa nyonic@ufs.ac.za

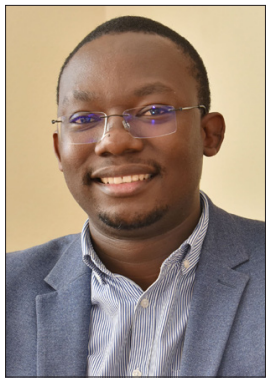

World Health Organization. State of the World's Nursing Report 2020: Investing in Education, Jobs and Leadership. Geneva: WHO, 2020

Trede F, Macklin R, Bridges D. Professional identity development: A review of the higher education literature. Stud High Educ 2012;37(3):365-384. https://doi.org/10.1080/03075079.2010.521237

. World Health Organization. Framework for Action on Interprofessional Education and Collaborative Practice. Geneva: WHO, 2010.

4. Botma Y, Labuschagne M. Students perceptions of interprofessional education and collaborative practice Analysis of freehand drawings. J Interprof Care 2019;33(3):321-327. https://doi.org/10.1080/13561820.2019.16 05981

5. Nyoni $\mathrm{CN}$, Botma Y. Implementing a competency-based midwifery programme in Lesotho: A gap analysis. Nurse Educ Pract 2019;34:72-78. https://doi.org/10.1016/.nepr.2018.11.005

Afr J Health Professions Educ 2020;12(4):165. https://doi.org/10.7196/AJHPE.2020.v12i4.1456 